\title{
PERITONEAL FOLD AROUND THE GALL BLADDER: A CASE REPORT SEEN IN CADAVERIC LIVER
}

Gokul Krishna Reddy Nune1, Minati Patra²

\section{HOW TO CITE THIS ARTICLE:}

Gokul Krishna Reddy Nune, Minati Patra. "Peritoneal Fold around the Gall Bladder: A Case Report Seen in Cadaveric Liver". Journal of Evolution of Medical and Dental Sciences 2015; Vol. 4, Issue 35, April 30; Page: 6153-6157, DOI: $10.14260 /$ jemds/2015/896

ABSTRACT: Anomalous peritoneal folds around the gallbladder are very rare and a few were reported in the past. The presence of such peritoneal folds is probably due to extension of the ventral mesentery which forms the lesser omentum.1,2,3 In the present case we report anomalous peritoneal fold suspending the gall bladder from the under surface of the liver, totally covering the gall bladder. The gall bladder with unusual peritoneal folds has a tendency to volvulus, a rare surgical disease which demands the immediate surgical intervention.2,3 Awareness of the possibility of such peritoneal anomaly around gallbladder facilitates the interpretation of image findings for proper diagnosis.

KEYWORDS: Gallbladder, peritoneal fold, volvulus, ventral mesentry.

INTRODUCTION: Peritoneum is the largest and most complexly arranged serous membrane. It lines the abdominal parities and is reflected over the viscera to invest them partially or completely. The peritoneal reflections form several folds which are called the ligaments, mesenteries and omenta. 1,2,3

These folds suspend and provide support to the abdominal organs and carry blood vessels to them. The peritoneal reflections divide the peritoneal cavity into recesses and spaces and form the boundaries to them. ${ }^{2}$ Specific attachments of these folds to the abdominal wall and viscera determine the route of spread of intra-peritoneal fluid and consequently, disease processes within the abdominal cavity. ${ }^{3}$ The peritoneal cavity and its reflections are the frequent sites for the infective, inflammatory, neoplastic and traumatic processes. In utero, the alimentary tract develops as a single tube suspended in the coelomic cavity by ventral and dorsal mesenteries.2,3 Ultimately, these mesenteries undergo complex process of development where various primitive mesenteries disappear. If for any reason, the primitive mesenteries persist they result in the formation of abnormal peritoneal folds. ${ }^{4,5}$ Presence of such folds around the gallbladder is very rare but a few cases have been reported in the past (Omer et al, 2006; Wendel, 1898). The gallbladder with unusual peritoneal fold has a tendency to volvulus (Shaikh et al, 2005).

CASE REPORT: The present case with the anatomical anomaly of peritoneum around the gallbladder was observed in a middle aged formalin embalmed female cadaver, during dissection classes of abdomen to the undergraduate medical students at Kalinga Institute of Medical Sciences Bhubhaneswar, India.

In this case gallbladder was found to be fully covered by the fold of peritoneum making the gall bladder invisible, the mesentry was attached to the gall bladder fossa. In the same liver the groove for the inferior venacava converted to tunnel by the formation of pons hepatis. 


\section{CASE REPORT}
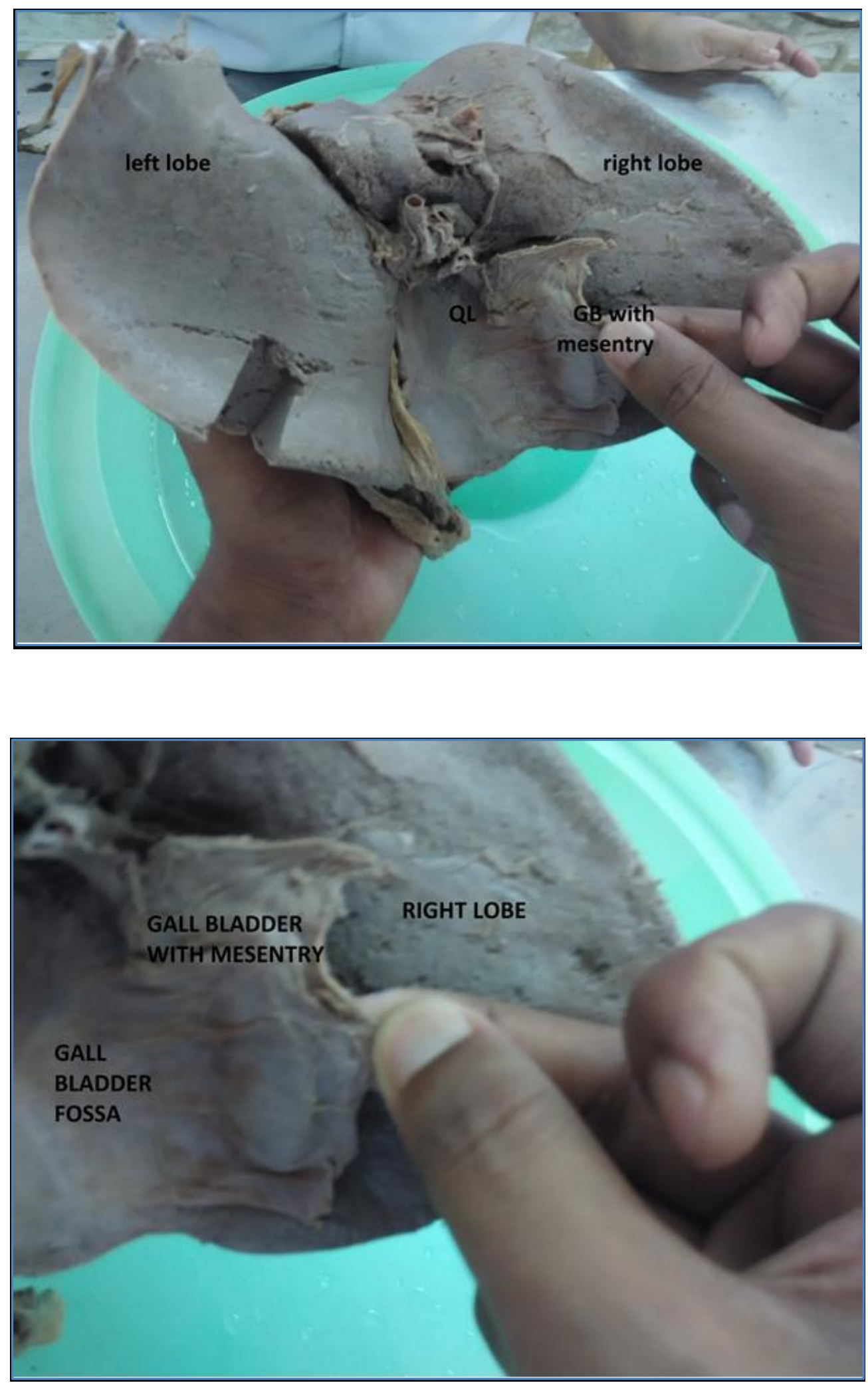


\section{CASE REPORT}

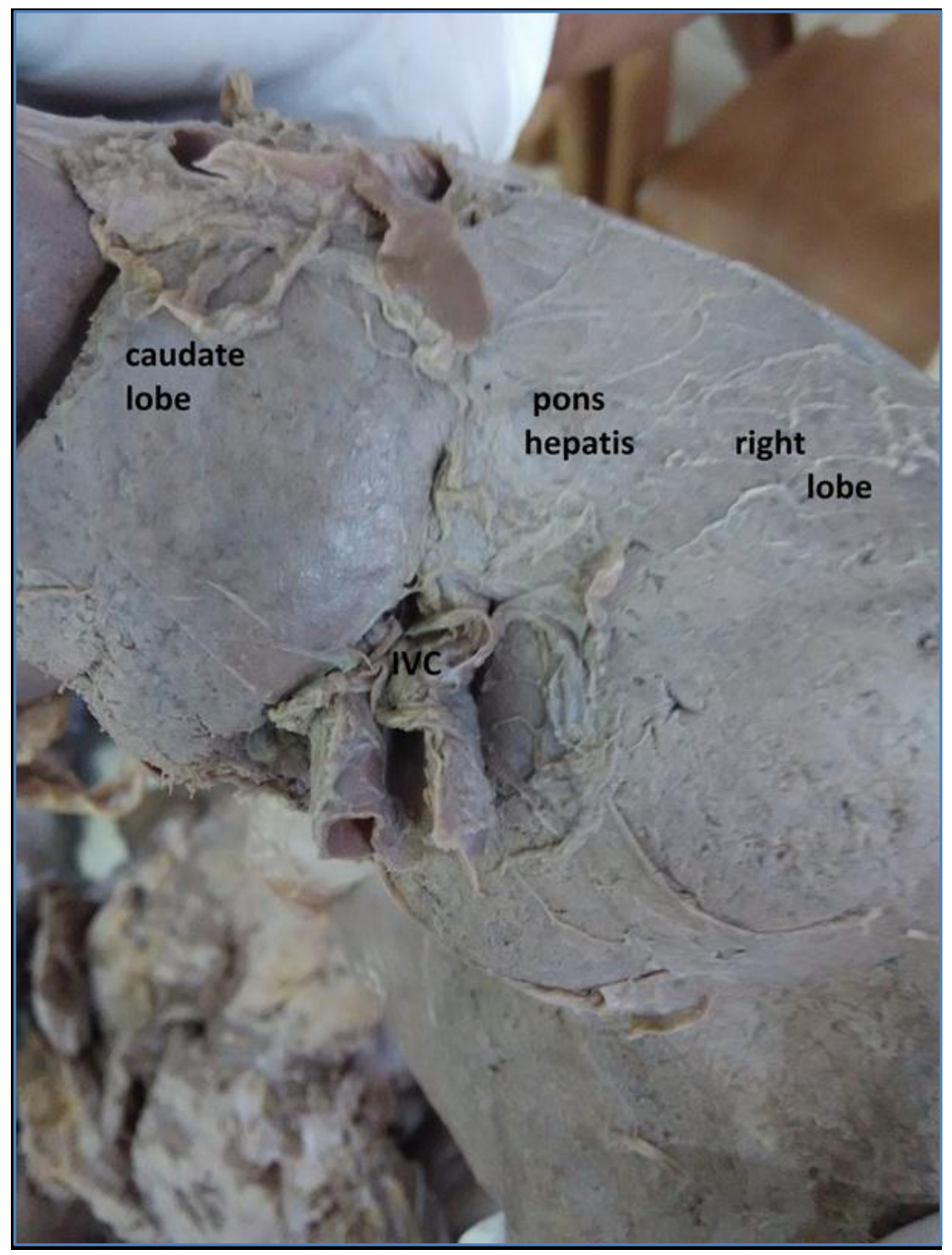

DISCUSSION: The rotation of the gallbladder on its mesentery along the axis of the cystic duct and cystic artery leads to gallbladder volvulus (Tarhan et al, 2006). Wendel was the first person, who described this extremely rare surgical disease (Wendel, 1898). The presence of mobile peritoneal fold is the prerequisite for the gallbladder volvulus. Earlier two cases of volvulus of the gallbladder have been reported by Shaikh et al (2005). Gallbladder possessing supporting membranes has been observed in 4 to $5 \%$ of specimens (Sheila, 1993). A cadaveric study conducted by Sridevi et al (2011) revealed the presence of abnormal mobile fold in $1 \%$ of cases.

The abnormal position of the gallbladder associated with the mobile peritoneal fold has been described. In a study of 27 patients, there were thirteen lumbar, nine pelvic and five iliac gallbladders (Tzardinoglou et al, 1996). The abnormal peritoneal folds connecting the gall bladder with the surrounding structures have been observed. Sridevi et al (2011) have reported the unusual peritoneal fold connecting the gallbladder, on the left to gastro pyloric junction and on the right to the hepatic flexure of the colon. 
The abnormal cystogastrocolic fold associated with atrophy of the gallbladder has been reported (Pamidi et al, 2008). A case of abnormal peritoneal fold connecting the greater omentum with the liver, gall bladder, right kidney and lesser omentum has been observed (Satheesha, 2009). The occurrence of these peritoneal folds attributed to the abnormal predisposition of the ventral mesentery in the embryonic life.

During embryonic life, the primitive gastrointestinal tract is anchored to the anterior and posterior abdominal walls by ventral and dorsal mesenteries respectively. As the development proceeds most of the ventral mesentery disappears except in the region of terminal part of the oesophagus, the stomach and the upper part of the duodenum and is derived from the septum transversum. Later with the growth of the liver into the mesenchyme of the septum transversum, the ventral mesentery divides into; the lesser omentum, that extends from the lower portion of the oesophagus, stomach and upper portion of the duodenum to the liver; the falciform ligament extending from the liver to the ventral body wall (Moore and Persaud, 2003). The abnormal peritoneal fold of gallbladder observed in the present case may due to the extension of the ventral mesentery which forms the lesser omentum as explained by Sheila (1993).

In the current case, this peritoneal fold extended from gall bladder to transverse colon, transverse mesocolon, and second part of duodenum. The probable reason for the existence of this fold is an embryonic adhesion between the ventral and dorsal mesenteries during rotation of the stomach (Nayak, 2009). Volvulus usually occurs in the small intestine, caecum, and sigmoid colon and rarely in the stomach. Gallbladder volvulus is a very rare disease and it requires immediate surgical intervention (Sheik et al, 2005). Gallbladder with two different types of unusual peritoneal folds has a tendency to volvulus; those with a wide mesentery and those in which the mesentery covers only the cystic duct and artery.

The peritoneal fold of gallbladder observed in the present case is unlikely to go for volvulus, but the loss of fat and atrophy of liver which occur with advancing age may lengthen this peritoneal fold and then it may lead to volvulus (McHenry and Byrne, 1986). The abnormal peritoneal fold around gallbladder can result in misinterpretation of imaging findings. In summary, the awareness of the possibility of such anomaly facilitates the proper diagnosis. The knowledge of abnormal peritoneal folds around the gallbladder and its attachments with surrounding structures is essentially important for the surgeons dealing with the surgeries of liver, gallbladder, duodenum and colon. It is also important for the surgeons as they should not mistake these folds for the adhesions induced by recurrent attacks of cholecystitis.

\section{REFERENCES:}

1. McHenry CR, Byrne MP. 1986. Gallbladder volvulus in the elderly. An emergent surgical disease. J Am Geriatr Soc 34: 137-139.

2. Moore KL, Persaud TVN. 2003. The Developing Human - Clinically oriented embryology. $7^{\text {th }}$ Ed Philadelphia. Saunders, 256-275.

3. Nayak BS. 2009. Abnormal peritoneal fold connecting the greater omentum with the liver, gallbladder, right kidney and lesser omentum.

4. Bratisl Lek Listy 110: 736-737. Pamidi N, Nayak S, Volllala VR. 2008.

5. Cystogastrocolic fold and associated atrophy of the gall Bladder. Singapore med J 49: 250. 


\section{AUTHORS:}

1. Gokul Krishna Reddy Nune

2. Minati Patra

\section{PARTICULARS OF CONTRIBUTORS:}

1. Post Graduate Student, Department of Anatomy, Kalinga Institute of Medical Sciences, Patia.

2. Professor, Department of Anatomy, Kalinga Institute of Medical Sciences, Patia.

\section{FINANCIAL OR OTHER} COMPETING INTERESTS: None

\section{NAME ADDRESS EMAIL ID OF THE} CORRESPONDING AUTHOR:

Dr. Gokul Krishna Reddy Nune, Department of Anatomy, Kalinga Institute of Medical Sciences, KIIT University Campus-5, Patia, Bhubhaneswar-751024, Odisha.

E-mail: gkr.nune@gmail.com

Date of Submission: 08/04/2015. Date of Peer Review: 09/04/2015. Date of Acceptance: 22/04/2015. Date of Publishing: 30/04/2015. 